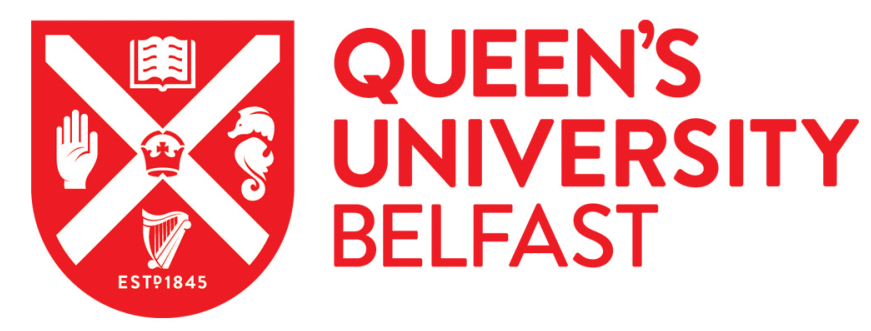

\title{
Electron rescattering in strong-field photodetachment of F-
}

Hassouneh, O., Law, S., Shearer, S. F. C., Brown, A. C., \& van der Hart, H. W. (2015). Electron rescattering in strong-field photodetachment of F-. Physical Review A (Atomic, Molecular, and Optical Physics), 91(3), [031404(R)]. https://doi.org/10.1103/PhysRevA.91.031404

Published in:

Physical Review A (Atomic, Molecular, and Optical Physics)

Document Version:

Peer reviewed version

Queen's University Belfast - Research Portal:

Link to publication record in Queen's University Belfast Research Portal

Publisher rights

Copyright 2015 APS

\section{General rights}

Copyright for the publications made accessible via the Queen's University Belfast Research Portal is retained by the author(s) and / or other copyright owners and it is a condition of accessing these publications that users recognise and abide by the legal requirements associated with these rights.

Take down policy

The Research Portal is Queen's institutional repository that provides access to Queen's research output. Every effort has been made to ensure that content in the Research Portal does not infringe any person's rights, or applicable UK laws. If you discover content in the Research Portal that you believe breaches copyright or violates any law, please contact openaccess@qub.ac.uk. 


\title{
Electron rescattering in strong-field photodetachment of $\mathrm{F}^{-}$
}

\author{
O. Hassouneh, ${ }^{1}$ S. Law ${ }^{1}$ S. F. C. Shearer, ${ }^{1}$ A. C. Brown, ${ }^{1}$ and H. W. van der Hart ${ }^{1}$ \\ ${ }^{1}$ Centre for Theoretical Atomic, Molecular and Optical Physics, \\ Queen's University Belfast, Belfast BT7 1NN, UK
}

(Dated: March 11, 2015)

\begin{abstract}
We present $a b$ initio studies of photoelectron spectra for above threshold detachment (ATD) of $\mathrm{F}^{-}$anions in short, $1300 \mathrm{~nm}$ and $1800 \mathrm{~nm}$ laser pulses. We identify and assess the importance of electron rescattering in strong-field photodetachment of a negative ion through comparison with an analytic, Keldysh-type approach, demonstrating the capability of ab-initio computation in the challenging near-IR regime. We further assess the influence of the strong electron correlation on the photodetachment.
\end{abstract}

PACS numbers: 32.80. Gc $31.15 . \mathrm{V}-$

Electron rescattering is one of the fundamental processes occuring in the interaction between matter and intense light fields [1]. The mechanism is a critical part of the well known three-step or recollision model for high harmonic generation (HHG) or strong field double ionisation. According to the model an electron is first ionised, then driven by a strong laser field, before recolliding with the parent ion, either recombining, leading to HHG $[2,3]$, or rescattering, leading to high-energy electron emission $[4,5]$, or non-sequential double ionisation [6].

Electron rescattering also encodes structural information about the residual ion into the wavepacket of the ejected electron and can thus be exploited as an experimental probe of the structure of the parent ion [1]. The technique is especially sensitive as the current density of a recolliding electron wavepacket exceeds that of conventional electron sources by several orders of magnitude [7]. Furthermore, the inherently subcycle and phase-locked nature of the recollision process gives access to electron dynamics on the attosecond scale, via information embedded in the photoelectron spectrum $[8,9]$.

One of the open questions in strong-field science concerns the importance of electron rescattering for negative ions. Significant progress has been made in understanding and controlling the equivalent process in neutral atoms and positive ions [10], but above-threshold detachment (ATD) presents a different challenge. The small binding energy allows detachment at low intensities. Hence to reach significant recollision energies, nearinfrared (NIR) laser fields are required. In addition, the absence of the Coulomb potential makes it easier for the electron wavepacket to spread out, reducing the effect of rescattering $[4,5]$. While evidence for rescattering from negative ions has been found experimentally [11], no verification has yet been provided from $a b$ initio theory. A theoretical approach, based on first order correction to the strong field approximation, was able to reproduce experimental results from $\mathrm{Br}^{-}$and $\mathrm{F}^{-},[12]$ but a more recent study, using a numerical solution of the time-dependent Schrödinger equation (TDSE), found "no qualitative evidence of rescattering" for $\mathrm{H}^{-}$[13]. In this report we demonstrate that ab-initio theory can be used to investigate rescattering in the NIR regime.

An additional complication in the description of negative ions is the much larger influence of dielectronicrepulsion. Several approximate methods have been employed to model photodetachment from complex negative ions $[13,14]$, but these methods are limited in their description of electron repulsion. A previous study found that multiphoton detachment rates for $\mathrm{F}^{-}$are affected substantially by the inclusion of correlation effects [15]. The accurate description of dielectronic repulsion may prove especially important in rescattering calculations for negative ions, as the neutral core means the process will be mediated entirely by short-range effects rather than the long-range potential of a charged core.

In this letter we address these fundamental questions by applying R-matrix theory with time-dependence (RMT) to study strong-field dynamics of $\mathrm{F}^{-}$in NIR laser pulses. The RMT method is an ab initio method for solving the TDSE for multielectron atomic systems in intense, short laser pulses. As with all R-matrix methods, it employs a division of space, whereby electron exchange effects are fully described in an inner-region close to the nucleus, while far from the nucleus, a single, ejected electron moves in the long-range multipole potential of the core. Although several other time-dependent R-matrix methods have emerged in recent years [16-18], RMT exhibits orders-of-magnitude improvements in efficiency, primarily because it employs finite-difference (FD) techniques to model the one-electron wave function in the outer region. RMT merges the outer region FD model with a B-Spline-based, R-matrix basis set for the multielectron inner region, allowing efficient calculations accounting for atomic structure and correlation effects [19].

The R-matrix basis for $\mathrm{F}^{-}$consists of the neutral $\mathrm{F}$ ground state coupled with an additional electron. We employ two different models for the F atom. These two models are compared to assess the influence of electron correlation. The basic model includes only one configuration, $1 s^{2} 2 s^{2} 2 p^{5}$, with the $1 s, 2 s$ and $2 p$ orbitals given by the Hartree-Fock orbitals for the F ground state [15, 20]. 

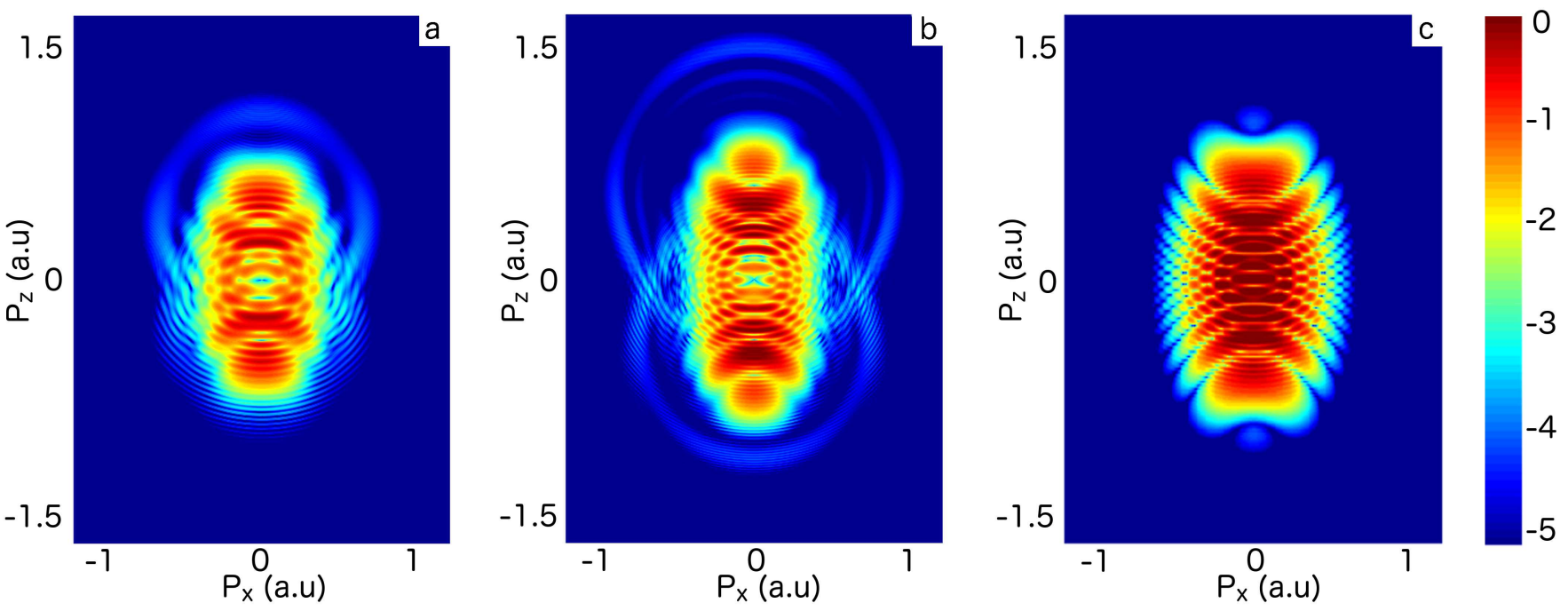

FIG. 1: (Color online) Electron momentum distributions for ATD of $\mathrm{F}^{-}$in the $k_{x}-k_{z}$ plane for a (a) $1300 \mathrm{~nm}(\mathrm{~b} / \mathrm{c}) 1800 \mathrm{~nm}$ laser pulse of intensity $1.3 \times 10^{13} \mathrm{~W} / \mathrm{cm}^{2}$. (a) and (b) are calculated using the RMT approach, while (c) is obtained using the KTA method [14].

The second model includes additional $\overline{3 s}, \overline{3 p}$, and $\overline{3 d}$ pseudoorbitals, [21]. This allows us to generate an accurate wave function expansion for the $1 s^{2} 2 s^{2} 2 p^{5}{ }^{2} P^{o}$ state from a configuration interaction calculation including the $1 s^{2} 2 s^{2} 2 p^{5}, 1 s^{2} 2 s 2 p^{5} 3 s, 1 s^{2} 2 s^{2} 2 p^{4} 3 p, 1 s^{2} 2 s^{2} 2 p^{3} 3 p^{2}$ and $1 s^{2} 2 s^{2} 2 p^{3} 3 d^{2}$ configurations. Model 2 gives a binding energy of $3.421 \mathrm{eV}$ for the ${ }^{1} S^{e} \mathrm{~F}$ ground-state, which is close to the experimental value of $3.399 \mathrm{eV}$ [22], whereas in model 1 we shift the ground-state artificially to $3.421 \mathrm{eV}$.

We employ $1300 \mathrm{~nm}$ and $1800 \mathrm{~nm}$ wavelength fields at a peak intensity of $1.3 \times 10^{13} \mathrm{Wcm}^{-2}$. The profile comprises two cycles $\sin ^{2}$ ramp-on followed by two cycles $\sin ^{2}$ ramp-off. In such high intensity, long-wavelength fields, the ejected electron wavepacket can travel far from the nucleus, and hence, an accurate description of the wave function is required over an extended region of space. To facilitate this we include angular momenta up to $L=240$ and propagate the wave function out to a radius of $4265 \mathrm{a}_{0}$. The time step in the wave function propagation is 0.24 as.

The laser parameters are chosen to facilitate a comparison with results obtained using a Keldysh-Type Approach (KTA) calculation [14, 23, 24]. In this model, the effect of the atomic potential on a detached electron is neglected. The description of $\mathrm{F}^{-}$is based on Hartree-Fock orbitals for $\mathrm{F}^{-}$in which the long range tail is fitted to the correct binding energy. The laser field is infinitely long, but with a periodic envelope which describes a series of short pulses. This allows the analytical solution of the so-called saddle-point equation, yielding the electron trajectories in the field. As a consequence of the long laser 'pulse', the field is composed of three distinct photon energies, which is at variance with our time-dependent R- matrix calculations wherein a spread of photon energies follows from the isolated short pulse used.

Figure 1 shows the $2 \mathrm{D}$ electron momentum spectra for ATD from $\mathrm{F}^{-}$for $1300 \mathrm{~nm}$ and $1800 \mathrm{~nm}$ fields alongside the KTA spectrum for $1800 \mathrm{~nm}$ [14]. The $z$-axis is directed along the polarization axis of the laser. The figure shows results from the basic atomic structure, model 1 of $\mathrm{F}^{-}$described above, which allows a closer comparison to the KTA results. The F atom has three different possible final states, corresponding to $m=0, \pm 1$ where $m$ is the magnetic quantum number. The total momentum distribution is obtained by incoherently summing the contributions from the $m=0$ and the $m= \pm 1$ states.

The ATD momentum distribution in Fig. 1 is extensively detailed. Both the RMT and KTA spectra show the typical structure of rings centered at zero momentum, with each ring corresponding to the absorption of $N$ photons. Interference patterns arise because multiple electron trajectories contribute to each final momentum state [13]. Lines connecting the interference minima are curves in momentum space which satisfy a destructive interference condition. Curves intersecting the $P_{z}$-axis correspond to interference between the well known longand short-trajectories. Curves intersecting the $P_{x}$-axis lead to minima/maxima in the even/odd ATD rings for perpendicular emission. The two different approaches therefore demonstrate the same basic physics of photodetachment. However, the features appear sharper in the KTA spectra due to the well defined photon energy, $\omega$, while for RMT the results are blurred by uncertainty in $\omega$. Furthermore, the RMT spectrum appears narrower in the $k_{x}$ direction.

Figure 1 shows additional ATD rings for electron mo- 
menta greater than the maximum drift momentum, $P_{z}=$ $\mathcal{E}_{0} / \omega$ (where $\mathcal{E}_{0}$ is the maximum strength of the electric field) for both $1300 \mathrm{~nm}$ and $1800 \mathrm{~nm}$ with the RMT calculations. These extra rings are not present in the KTA model results. In the positive $z$ direction, the rings are centred on $P_{z} \approx 0.55$ a.u., and $P_{z} \approx 0.75$ a.u. for $1300 \mathrm{~nm}$ and $1800 \mathrm{~nm}$ respectively: these values corresponding to the maximum drift momentum for each wavelength.

These additional rings are a clear signature of electronrescattering. In the strong field electrons are detached near the maximum of the field each half cycle as per the three-step model. The electrons are thus 'born' into the field with zero energy, leading to ATD rings centered on zero momentum as in Fig. 1. Recollision then occurs near the zero-field intersections, allowing the electron to gain extra drift momentum through its interaction with the field potential. Rescattered electrons are effectively 'reborn' into the field at the field-zero, gaining a maximum drift momentum of $P_{z}=\mathcal{E}_{0} / \omega$. This can be confirmed with classical trajectory calculations [25]. The appearance of these rings is proof of the recollision mechanism in negative ions. Angle resolved spectra showing these rings centered on the drift momentum go beyond the integrated yields shown in Fig. 2 and correspondingly in [12], which show only evidence of high energy electrons which are ascribed to the recollision mechanism.

The results display a clear asymmetry in the $P_{z}$ direction, with the recollision rings in the positive $z$ direction extending to larger momenta than in the negative $z$ direction. This is due to the short pulse profile we employ. The highest energy electrons are accelerated by the single, peak-intensity cycle of the laser pulse and hence are only emitted in the positive direction. Moreover, in the negative $z$ direction, there is a pronounced interference structure in the recollision rings, arising from two interfering electron trajectories in the ramp-on and ramp-off of the field. Finally, the centre of these recollision rings is shifted towards the origin for the negative $z$ direction, because the electron trajectories in the ramp-on and rampoff of the field are driven by lower intensity laser cycles. Thus the centre of the recollision rings in the negative $z$ direction is given by $0.85 \times \mathcal{E}_{0} / \omega$. We note that the non-recollision part of the momentum distribution is mediated by the vector potential of the laser field, which is antisymmetric about zero potential. Thus this part of the distribution is symmetric with respect to the origin, while the recollision, mediated by the asymmetric electric field, leads to an asymmetric spectrum. We would expect the opposite to be true, were the carrier envelope phase of the laser pulse adjusted accordingly.

A second outstanding question surrounding strongfield dynamics of negative ions concerns the influence of electron correlation. Since the outer electron in $\mathrm{F}^{-}$is loosely bound, and the main rescattering process occurs very close to the residual atom, electron correlation may affect both the initial ionization and the rescattering. A
TABLE I: The level of ionisation of $\mathrm{F}^{-}$in $1300 \mathrm{~nm}$ and $1800 \mathrm{~nm}$ fields calculated for different peak intensities. This ionised population is presented for the two models used in RMT, compared to the total detachment probability from [14]

\begin{tabular}{lccc}
\hline \hline $\begin{array}{c}\text { Intensity } \\
\mathrm{W} / \mathrm{cm}^{2}\end{array}$ & \multicolumn{3}{c}{ Detachment probability } \\
\hline \multicolumn{4}{c}{$1300 \mathrm{~nm}$} \\
\hline $7.7 \times 10^{12}$ & 0.018 & 0.011 & 0.036 \\
$1.1 \times 10^{13}$ & 0.045 & 0.031 & 0.090 \\
$1.3 \times 10^{13}$ & 0.065 & 0.044 & 0.139 \\
\hline \multicolumn{5}{c}{$1800 \mathrm{~nm}$} \\
\hline $7.7 \times 10^{12}$ & 0.020 & 0.013 & 0.023 \\
$1.1 \times 10^{13}$ & 0.055 & 0.034 & 0.066 \\
$1.3 \times 10^{13}$ & 0.080 & 0.052 & 0.106 \\
\hline
\end{tabular}

recent study suggests that while for neutral atoms there are notable differences between KTA model results and those from the numerical solution of the TDSE, these differences are negligible for negative ions [13]. The ejected-electron momenta spectra obtained for model 1 and model 2 show little qualitative difference, but notable, quantitative differences can be observed when electron correlation is included.

Table I gives the population in the outer region- a measure of detachment probability in our calculationscompared with the detachment probability from the KTA model for $1300 \mathrm{~nm}$ and $1800 \mathrm{~nm}$. The yield in our model 1 calculations is reduced by a factor of 2 from the KTA model result for $1300 \mathrm{~nm}$, whereas the difference is only about $20 \%$ for $1800 \mathrm{~nm}$. When using the more sophisticated model 2 for $\mathrm{F}^{-}$, we find a further reduction in the detachment probability by one third. The reason for these differences is not obvious, although the effective potentials differ. The binding energy in RMT model 1 is shifted artificially to the experimental value, while model 2 gives the correct binding energy directly. This implies that the short-range potential, and therefore the wave function, is described more accurately in model 2. This increase in accuracy may lead to detachment yields about a third smaller than model 1 , and a factor of 2-3 smaller than the KTA. Differences in short-range potential can thus lead to significant differences in detachment yield.

The differences between model 1 and model 2 are most easily identified in the photelectron energy spectra, which are presented in Figs. 2 and 3 alongside results from the KTA model. In all calculations, model 1 shows a larger electron yield across the full energy range, reflecting the lack of change in the overall structure of the spectrum. The figures show that the main effect of electron interaction is an overall reduction in detachment probability.

Figure 2 demonstrates the capability of the RMT approach to describe electron rescattering. For energies up to about $10 \mathrm{eV}$, the RMT and KTA calculations show a 

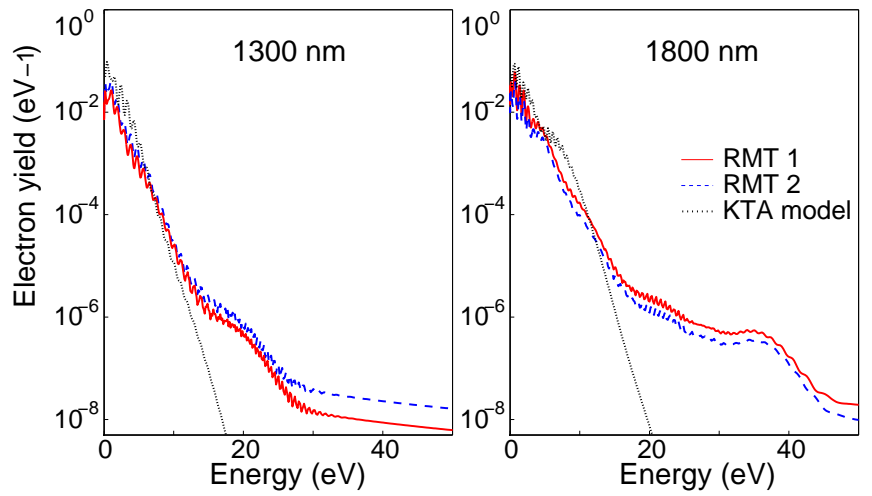

FIG. 2: (Color online) Photoelectron energy spectra for ATD from $\mathrm{F}^{-}$by a $1.3 \times 10^{13} \mathrm{~W} / \mathrm{cm}^{2}$ pulse at $1300 \mathrm{~nm}$ and $1800 \mathrm{~nm}$ from the present approach, model 1 (solid, red line), model 2 (dashed, blue line) and the KTA model approach (dotted, black line). The presence of 'knee'-like structures in the RMT results is a characteristic signature of rescattering.

very similar behaviour for electron emission, consisting of a small initial plateau followed by rapid exponential decay. Differences emerge for energies greater than about $10 \mathrm{eV}$, with the RMT calculations showing a plateau at a magnitude of $\sim 10^{-5}-10^{-6} \mathrm{eV}^{-1}$ extending to an energy of about $20 \mathrm{eV}$ for $1300 \mathrm{~nm}$ and $35 \mathrm{eV}$ for $1800 \mathrm{~nm}$, corresponding to around $10 U_{p}$ in each case. This plateau is absent in the KTA calculations. Since the KTA approach does not account for rescattering we can identify this as the source of the plateau. The yields obtained in model 1 and model 2 are very similar, with those obtained in model 2 lying about one third lower than for model 1 .

Figure 3 shows the low energy region of the photoelectron spectra, which display peaks characteristic of the multiphoton mechanism: each peak corresponding to the absorption of an integer number of photons and with energy, $E=N \omega-U_{P}-I_{P}$ for some integer $N$, and the ionization energy, $I_{p}$. In all cases, the magnitude of the photoelectron peaks is lower for the present calculations than for the KTA approach and lower for model 2 than for model 1 , consistent with the lower detachment probability discussed above. The qualitative agreement with the KTA model is excellent for $1800 \mathrm{~nm}$, while, at $1300 \mathrm{~nm}$, the positions of the ATD peaks differ. The origin of this difference is difficult to assess unambiguously. However, two factors may play a role. Firstly, the RMT calculations account for AC-Stark shifts beyond the ponderomotive energy shift, whereas the KTA model includes the ponderomotive shift only. Secondly, the RMT method uses a short laser pulse, while the KTA uses an infinitely long field with a periodic envelope. This leads to differences in the photon energy and consequently the ponderomotive shift, making it difficult to determine the correct position of the photoelectron peaks. It will therefore be interesting to compare these results with experimental spectra, although, as far as we are aware, only

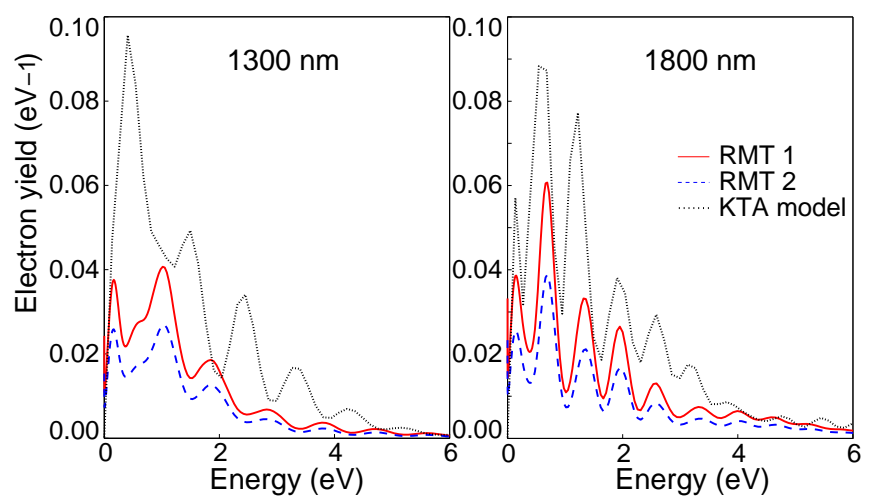

FIG. 3: (Color online) As Fig. 2 but shown for the low energy region on a linear scale.

on-axis emission has been measured for the present laser parameters [26].

In conclusion, we have demonstrated the capabilitiy of $a b$ initio theory to study ATD of $\mathrm{F}^{-}$anions in computationally challenging NIR laser pulses with a full description of multielectron effects. Through comparison with a KTA model we have identified the importance of the recollision mechanism in the electron momentum distributions. High energy rings in the angle resolved photoelectron spectra are the first theoretical verification of rescattering from negative ions. The integrated photoelectron energy spectra shows further clear evidence of rescattering, with a knee-like, plateau structure extending to energies of $10 U_{p}$. Although the yield in these rescattering channels is small- on the order of .02\% and .07\% of the total yield for $1300 \mathrm{~nm}$ and $1800 \mathrm{~nm}$ respectively- it is clear evidence of rescattering.

The description of multielectron atoms and ions in short, intense NIR pulses is a significant computational challenge, but holds substantial promise for elucidating new physics, mediated by multielectron interaction. The RMT method has already been applied to other strong field phenomena, including high-harmonic generation [27], but the accurate and efficient determination of the multielectron wave function over such a large region of space is a first for calculations of this type. Indeed, the present calculation of ATD in negative ions provides an extremely sensitive test of the wave function accuracy. It will be interesting now to compare theoretical single-atom results of the RMT approach with the findings of state-of-the-art experimental techniques in strongfield physics and model approaches [12].

$\mathrm{OH}$ acknowledges financial support from the University of Jordan. HWH acknowledges financial support from the UK EPSRC under grant no. EP/G055416/1 and the EU Initial Training Network CORINF. SL is funded by DEL-NI under the programme for government. This work used the ARCHER UK National Supercomputing Service (http://www.archer.ac.uk). The data 
used in this paper may be accessed via pure.qub.ac.uk/ portal/en/datasets/search.html.

[1] P. B. Corkum and F. Krausz, Nat. Phys. 3, 381 (2007).

[2] P. B. Corkum, Phys. Rev. Lett. 71, 1994 (1993).

[3] M. Lewenstein, P. Balcou, M. Y. Ivanov, A. L'Huillier, and P. B. Corkum, Phys. Rev. A. 49, 2117 (1994).

[4] H. W. van der Hart and K. Burnett, Phys. Rev. A 62, 013407 (2000).

[5] G. L. Yudin and M. Y. Ivanov, Phys. Rev. A 64, 035401 (2001).

[6] D. N. Fittinghoff, P. R. Bolton, B. Chang, and K. C. Kulander, Phys. Rev. Lett. 69, 2642 (1992).

[7] H. Niikura et al., Nature 417, 917 (2002).

[8] C. I. Blaga et al., Nature 483, 194 (2012).

[9] F. Lépine, M. Y. Ivanov, and M. J. J. Vrakking, Nat. Photon. 8, 195 (2014).

[10] D. B. Milošević, G. G. Paulus, D. Bauer, and W. Becker, J. Phys. B: At. Mol. Opt. Phys. 39, R203 (2006).

[11] J. Pedregosa-Gutierrez et al., Phys. Rev. Lett. 93, 223001 (2004).

[12] A. Gazibegović-Busuladžić et al., Phys. Rev. Lett. 104, 103004 (2010).

[13] P. A. Korneev et al., New J. Phys. 14, 055019 (2012).
[14] S. F. C. Shearer and M. R. Monteith, Phys. Rev. A 88, 033415 (2013)

[15] H. W. van der Hart, J. Phys. B: At. Mol. Opt. Phys. 33, 1789 (2000).

[16] H. W. van der Hart, M. A. Lysaght, and P. G. Burke, Phys. Rev. A 76, 043405 (2007).

[17] X. Guan, C. J. Noble, O. Zatsarinny, K. Bartschat, and B. I. Schneider, Phys. Rev. A 78, 053402 (2008).

[18] M. A. Lysaght, P. G. Burke, and H. W. van der Hart, Phys. Rev. Lett. 101, 253001 (2008).

[19] L. R. Moore et al., J. Mod. Optics 58, 1132 (2011).

[20] H. W. van der Hart, J. Phys. B: At. Mol. Opt. Phys. 29, 3059 (1996).

[21] C. Niang and M. Dourneuf (1997), unpublished.

[22] R. S. Berry and C. W. Reimann, J. Chem. Phys. 38, 1540 (1963).

[23] G. F. Gribakin and M. Y. Kuchiev, Phys. Rev. A 55, 3760 (1997).

[24] S. F. C. Shearer, M. C. Smyth, and G. F. Gribakin, Phys. Rev. A 84, 033409 (2011).

[25] K. Varjú et al., J. Phys. B: At., Mol. Opt. Phys. 39, 3983 (2006).

[26] I. Y. Kiyan and H. Helm, Phys. Rev. Lett. 90, 183001 (2003).

[27] O. Hassouneh, A. C. Brown, and H. W. van der Hart, Phys. Rev. A 90, 043418 (2014). 